AIMS Genetics, 6(2): 11-13.

AIMS Genetics

DOI: 10.3934/genet.2019.2.11

Received: 31 May 2019

Accepted: 31 May 2019

Published: 3 June 2019

http://www.aimspress.com/journal/Genetics

\title{
Highlight
}

\section{Linking metabolic and epigenetic regulation in the development of lung cancer driven by TGF $\beta$ signaling}

\author{
Liyi Zhang*
}

Sun Yat-sen University Cancer Center, 651 Dongfeng Road East, Guangzhou 510060, P. R. China

* Correspondence: Email:zhangly1@sysucc.org.cn.

Abbreviations: FH: Fumarase; NICD: Notch intracellular domain

In addition to the basic role in metabolic pathways, metabolic enzymes in cancer cells also actively participate in non-metabolic activities through related metabolic activity [1], such as epigenetic regulation [2]. Fumarase (FH) is one of the most important metabolic enzymes in the tricarboxylic acid cycle. It involved in the reversible hydration and dehydration of fumarate to malate. Previous study has shown that Fumarate can compete with $\alpha$-ketoglutarate to bind to $\alpha$-ketoglutarate-dependent dioxygenases (the enzymes that are involved in histone and DNA demethylation) and thereafter exert an inhibitory effect [3]. FH deficiency promotes the development of renal cancer by inhibiting TET-regulated DNA demethylation [4]. FH is also reported to regulate H3K36me2 at the region of DNA breaks or gene promoter by antagonizing $\alpha$ - ketoglutarate -dependent demethylase (KDM2A/B) in glucose-deficient conditions [5]. In view of the close relationship between the functional status of $\mathrm{FH}$ and epigenetic regulation, it is very meaningful to understand the specific role of FH in cancer development and progression.

TGF $\beta$ has been implicated as a potent driver in tumor initiation and progression. It has been a hot issue to decipher how the functional role of TGF $\beta$ is converted. Emerging evidence in recent years indicates that TGF $\beta$ can induce cellular apoptosis or epithelial-to-mesenchymal transition through activating p38 and Notch pathway [6,7]. However, how its effects are coordinated remains elusive. A recent study reported that CSL, a central effector regulated by Notch pathway, inhibited cell senescence through its inhibitory effects on p53-mediated p21 expression [8]. This observation suggests that the regulation of p53 by CSL is a potential link between TGF $\beta$ and Notch signaling.

In a study recently published in Cancer Research, titled "PAK4 Phosphorylates Fumarase and Blocks TGF $\beta$-Induced Cell Growth Arrest in Lung Cancer Cells”, Chen et al. [9] uncovered a novel 
mechanism for the regulation of H3K36me2 by p38/FH axis, and demonstrated that the metabolic effect of FH on transcriptional regulation is closely related to the cellular response to TGF $\beta$ signaling in lung cancer.

To determine whether $\mathrm{FH}$ is involved in the regulation of TGF $\beta$ signaling under Notch activation, the authors examined the potential relation between FH and CSL in human bronchial epithelial cell line using immunoprecipitation analysis. They found that TGF $\beta$ stimulation dramatically increased the interaction between FH and CSL in bronchial epithelial cells expressing Notch intracellular domain (NICD). These results suggest that both TGF $\beta$ and Notch signaling are required for FH-CSL complex formation. Further flow cytometry analyses showed that FH was required for TGF $\beta$ induced growth arrest upon Notch activation. In addition, coimmunoprecipitation and human FH amino acid sequence analyses indicated that TGF $\beta$-induced FH-CSL interaction was dependent on p38 activity and Thr 90 is the potential p38-phosphorylated residue as S/TP site. Next, the author found that nuclear NICD promotes the interaction between CSL and p38-phosphorylated FH and thus FH/CSL/p53/Smad complex formation upon Notch activation. Additionally, NICD translocation into the nucleus facilitates $\mathrm{FH}$ recruitment to p53-targed p21 promoter, where $\mathrm{FH}$ inhibits KDM2A-mediated demethylation of H3K36me2 through local production of fumarate, whereas, accumulation of H3K36me2 relieves the inhibitory effect of CSL on p53 and promotes TGF $\beta$-induced cell growth arrest.

Previous study reported that FH localized in the nucleus displayed a local effect on histone methylation [10]. Although the supporting evidence for this study can only indirectly prove that the promoter-associated FH promotes p53-mediated transcription by inhibiting H3K36me2 demethylation, this study still provide crucial information to better understand the local metabolic effects of FH under the specific context and facilitate the development of novel experimental methods that can precisely detect the amount of local metabolites.

In addition to cell growth inhibition, TGF $\beta$ is known to drive tumor metastasis paradoxically. Interpreting the functional transformation of TGF $\beta$ is a hot topic. In this study, the authors found that TGF $\beta$-induced FH-CSL interactions and cell growth arrest were significantly inhibited in lung cancer cells, which were reversed by PAK4 inhibition. Moreover, PAK4 is highly expressed in lung cancer cells and clinical samples. It can phosphorylate $\mathrm{FH}$ at Ser 46 and inhibit the nucleus translocation of FH. In turn, FH Ser 46 phosphorylation is blocked with TGF $\beta$-induced Cell growth arrest was associated with nuclear function of FH. Taken together, this study suggest that abnormal levels of PAK4 will be an important strategy for cancer cells to avoid the negative effects of TGF $\beta$ on cell growth and thus indirectly enhance tumor metastasis under TGF $\beta$ signaling. This finding potentially provides a molecular basis for improving the clinical treatment against tumors with upregulated PAK4 activity.

\section{Conflict of interest}

The authors declare no conflict of interest.

\section{References}

1. Lu S, Wang Y (2018) Nonmetabolic functions of metabolic enzymes in cancer development. Cancer Commun 38: 63.

2. $\quad$ Wang Y, Lei Q (2018) Metabolic recoding of epigenetics in cancer. Cancer Commun 38: 25. 
3. Xiao M, Yang H, Xu W, et al. (2012) Inhibition of alpha-KG-dependent histone and DNA demethylases by fumarate and succinate that are accumulated in mutations of FH and SDH tumor suppressors. Genes Dev 26: 1326-1338.

4. Sciacovelli M, Goncalves E, Johnson TI, et al. (2016) Fumarate is an epigenetic modifier that elicits epithelial-to-mesenchymal transition. Nature 537: 544-547.

5. Wang T, Yu Q, Li J, et al. (2017) O-GlcNAcylation of fumarase maintains tumour growth under glucose deficiency. Nature Cell Biol 19: 833-843.

6. Horowitz JC, Lee DY, Waghray M, et al. (2004) Activation of the pro-survival phosphatidylinositol 3-kinase/AKT pathway by transforming growth factor-beta1 in mesenchymal cells is mediated by p38 MAPK-dependent induction of an autocrine growth factor. J Biological Chem 279: 1359-1367.

7. Timmerman LA, Grego-Bessa J, Raya A, et al. (2004) Notch promotes epithelial-mesenchymal transition during cardiac development and oncogenic transformation. Genes Dev 18: 99-115.

8. Procopio MG, Laszlo C, Al Labban D, et al. (2015) Combined CSL and p53 downregulation promotes cancer-associated fibroblast activation. Nature Cell Biol 17: 1193-1204.

9. Chen T, Wang T, Liang W, et al. (2019) PAK4 Phosphorylates Fumarase and Blocks TGFbeta-Induced Cell Growth Arrest in Lung Cancer Cells. Cancer Res 79: 1383-1397.

10. Jiang Y, Qian X, Shen J, et al. (2015) Local generation of fumarate promotes DNA repair through inhibition of histone H3 demethylation. Nature Cell Biol 17: 1158-1168.

\section{AIMS AIMS Press}

(C) 2019 the Author(s), licensee AIMS Press. This is an open access article distributed under the terms of the Creative Commons Attribution License (http://creativecommons.org/licenses/by/4.0) 